\title{
Research on Key Technology of Signal Control Subarea Partition Based on Correlation Degree Analysis
}

\author{
Huihui Lan ${ }^{1}$ and Xianyu Wu $\mathbb{D}^{2}$ \\ ${ }^{1}$ Zhejiang Provincial Institute of Communications Planning, Design \& Research, Xixi Garden, 928 Yuhangtang Road, \\ Xihu District, Hangzhou 310031, Zhejiang, China \\ ${ }^{2}$ Beijing Jiaotong University, School of Traffic and Transportation, \\ Beijing Jiaotong University No. 3 Shangyuancun Haidian District, Beijing 100044, China \\ Correspondence should be addressed to Xianyu Wu; wuxy@bjtu.edu.cn
}

Received 1 September 2019; Revised 2 February 2020; Accepted 11 February 2020; Published 16 March 2020

Academic Editor: Krzysztof Okarma

Copyright ( $\odot 2020$ Huihui Lan and Xianyu Wu. This is an open access article distributed under the Creative Commons Attribution License, which permits unrestricted use, distribution, and reproduction in any medium, provided the original work is properly cited.

\begin{abstract}
In order to accurately divide the traffic control subarea and improve the regional coordination control effect, this paper studies the key technologies of traffic control subarea division from the influence of the discrete characteristics of the fleet on the coordinated control. Correlation analysis method and regression analysis method are used to construct the correlation model of adjacent intersections. The principle and process of transportation subarea division are designed by using analytic hierarchy process, and a new traffic subarea division model is proposed. Taking Gulou Street in Miyun District of Beijing as an example, combined with the green wave coordination optimization method, the new model was applied and simulated in SimTraffic. The simulation results show that the average delay of the intersection is reduced by $21.4 \%$, the total number of stops is reduced by $9.62 \%$, and the travel time is shortened by $8.75 \%$. The new intersection degree model and the coordinated control scheme obtained by the traffic subarea division model make the highest traffic efficiency. That is, to verify the scientific research method of this paper, the research results are reliable and the practical application value is high.
\end{abstract}

\section{Introduction}

In urban traffic signal control, optimization of the overall urban road network signal control scheme will be a very complicated process due to the limitations of the signal controller's own capabilities and calculation time. Therefore, several intersections and road sections with similar traffic characteristics are classified into a series of control subnetworks, and then the signal control parameters of each control subarea network are optimized, respectively. Finally, the optimization results of each subarea are integrated. That is, the transformation of complex large-scale traffic signal control problems into small-scale signal control problems through network segmentation will greatly improve the optimization efficiency and capability of signal control schemes [1]. In other words, driving on a road controlled by continuous signals, if the intersection can be accurately divided into different traffic control subareas and adopting coordinated control methods, road traffic efficiency will be greatly improved, traffic congestion will be alleviated, energy consumption will be saved, and economy will be reduced loss. However, with the rapid development of urbanization, urban traffic conditions are becoming more and more complex. The early traffic control subarea division method has not applied the current traffic conditions.

Intersection correlation research is the basis of traffic control subarea division. For the study of the relevance of intersections, scholars at home and abroad have proposed a number of relevance models. In foreign countries, Yagoda [2] defines the coupling degree of road segment flow and link length to quantify the correlation of adjacent intersections and uses coupling degree to judge whether adjacent intersections need coordinated control; Chang [3] proposes downstream intersections. The arrival of the team is the 
decisive factor in determining whether the adjacent intersections need to be coordinated. The correlation model is established according to the flow ratio of the upstream intersection and the dispersion of the fleet. The 1987 edition of the US Capacity Manual [4] recommends the Whitson model. The model considers the travel time of the road segment and the traffic at the intersection; the NAC Traffic Control Device Instruction Manual [5] defines the correlation between the travel time of the vehicle traveling between adjacent intersections and the signal period of the intersection. That is, when the degree of association is less than 1 , it is considered that the adjacent intersection needs to be divided into one traffic control subzone for coordinated control. There are also many scholars in China who have studied the relevance model, such as the intersection correlation calculation model proposed by $\mathrm{Li}$ [6] of Tsinghua University. The calculation model of the adjacent intersection degree is proposed by Lu [7] of South China University of Technology. Duan [8] from Tsinghua University proposed the intersection degree PF. The path correlation degree is proposed by Yang [9] and others from Southeast University to characterize the quantitative correlation degree. Xu [10] of South China University of Technology proposed the correlation degree model in the high saturation state as the road segment queuing ratio. There are also correlation models proposed by Lin [11] and Zeng [12]. Although the current relevance model can derive the basic association degree for the subdivision model service, however, the current correlation degree model research is based on theoretical analysis, and there is no actual data support. At the same time, most models have many constraints, and there are some shortcomings such as complicated calculation and difficult data acquisition.

For the study of the division of traffic control subareas, Walinchus [13] first proposed the concept of traffic control subarea and defined the boundary of the traffic control subarea in the section where the flow or road characteristics changed significantly. FHWA [14] suggested using the spacing of adjacent intersections as the index. When the spacing of adjacent intersections is greater than 800 meters, it should not be classified into the same traffic control subarea. According to the coupling degree division method proposed by Yagoda [2], when the degree of coupling between adjacent intersections is greater than 0.5 ; it should be divided into a traffic control subarea for signal coordination control. The traffic control subarea division in the SCATS system [15] is to manually set the initial partition road network parameters with reference to specific principles, models, and algorithms. Zhao [16] analyzed the queuing characteristics of the road segment and proposed the ratio of the maximum traffic queue length to the length of the link as the subarea. Zhao [17] proposed a traffic subarea partitioning method based on GN algorithm. Zhao [17] proposed subarea division based on the degree of correlation of key intersections. Xia et al. [18] proposed a MapReduce-based parallel three-phase K-Means algorithm for large-flow data partitioning. Xie [19] established a fuzzy C-means clustering method. The theoretical research on the early subarea division method mainly stays at the level of static qualitative analysis and lacks comprehensive quantitative analysis of the relevant factors affecting adjacent intersections. At the same time, the dynamic traffic subarea division technology is still not mature. The model parameters of the traffic subarea dynamic partitioning algorithm are subjective factors with large interference. Most of them are under the assumption of conditions, lacking actual traffic data support, and the division results cannot be guaranteed to match the actual traffic conditions, affecting the actual operation of the coordinated control system.

In view of this, the research in this paper collected the traffic data of the fleet running at the late peak of Zhongxing Road in Jiangdong District, Ningbo City, Zhejiang Province, China, and comprehensively analyzed the fleet data, focusing on the discreteness of the fleet, establishing the correlation model and the traffic subdivision model. At the same time, the traffic data of Gulou Street in Miyun District, Beijing, China was collected. The research results of this paper were applied to Gulou Street, and the green wave was optimized by using the developed green wave bandwidth optimization method BOTSD [20]. SimTraffic is the most widely used and the least error traffic simulation software, so this paper chooses this software to simulate and evaluate the urban road conditions. Due to the formation of the fleet at the intersection of Zhongxing Road at the peak of the evening, there is no blockage and it is defined as moderate saturation, so the research results of this paper are applicable to moderately saturated intersections.

\section{Data Collection}

2.1. Relevance and Correlated Quantities Definition. This paper focuses on the discreteness of the platoon, which is to study the discrete changes of the platoon between adjacent intersections. It was observed that the longer the time interval between the front and rear vehicles passing through the intersection stop line, the more the platoon is dispersed, and the shorter the time interval is, the closer the platoon remains. The time interval is defined as the headway distance, and the average headway distance of the platoon is the arithmetic mean of the distance between the heads of each adjacent two vehicles in the platoon. Obviously, when the platoon travels to the downstream intersection, if the average headway distance becomes larger, the platoon becomes more discrete and the correlation of adjacent intersections is weaker. If the average headway distance becomes smaller, the closer the team maintains, the stronger the correlation of adjacent intersections is. Therefore, the originality of this paper proposes to characterize the correlation between adjacent intersections by the difference of the average headway distance between the adjacent intersections and define the difference between the average headway distances of the upstream and downstream intersections as adjacent intersection relevance $R$.

Relevance degree of adjacent intersection refers to the traffic and physical connection between adjacent intersections. Traffic characteristics include number of vehicles passing through adjacent intersections and sections and vehicle speed change. The degree of correlation determines 
whether adjacent intersections need coordinated control. The intersection-related traffic flow refers to the total flow entering the road section from the upstream intersection, including the southbound through movement, westbound left-turn movement, and eastbound right-turn movement, as shown in Figure 1(a). The link-related traffic flow refers to the traffic flow of the northbound entrance of the downstream intersection, which is the associated traffic of the two sections before and after the intersection, as shown in Figure 1(b). The length of the road section is the distance of the southbound stop-line between the upstream intersection and the downstream intersection.

2.2. Data Collection. Content of data collection is mainly divided into two parts, one is the platoon data, and the other is the related factors that affect the shape change of the platoon, including the link associated traffic, intersection associated traffic, link distance, signal timing plans and channelization plans, and movement turning flows (lane number, lane width, etc.) of intersections.

The practical platoon data is collected by video recorded for all the intersections at the same time. The specific position and orientation of the cameras on the road segment are shown in Figure 2(a). Then, the time of the vehicle continuously passing through the adjacent intersection stop line, the length of the platoon, and the number of vehicles from other noncritical intersections entering the platoon are obtained from the videos. The data collection time is from 5 $\mathrm{pm}$ to $7 \mathrm{pm}$ at the peak hours in the evening. The research area includes five consecutive intersections from Tongtu Road to Zhongshan East Road, as shown in Figure 2(b).

\section{Model Building}

3.1. Relevance Model. The correlation analysis method is used to analyze and process the survey data, and the irrelevant variables are eliminated. Only the most relevant quantity, the length of the link and the associated flow of the link are remained, and then the regression analysis is used to obtain a simple formula. The simple relevance model is as follows:

$$
R=\frac{1}{1+|2.6-0.06 q+0.002 l|} .
$$

In the abovementioned formula, $q$ is the associated traffic of the link in one cycle length, the unit is $\mathrm{pch} / \mathrm{C}$; and $l$ is the length of the link, in meters. In this model, the larger the associated traffic volume of the road segment, the shorter the length of the road segment and the greater the degree of association between adjacent intersections, which is more suitable for coordinated control.

3.2. Traffic Subarea Partition Model. The application of the model in the actual traffic subarea partition is mainly divided into two parts: one part is the model basis for forming the initial subplan, and this model is defined as the subarea division principle model. In another part, the boundary of the traffic subarea is determined based on the initial subplan, and then the model basis of the optimal division scheme is formed with the proposed association degree division model.

\subsubsection{Traffic Subarea Partition Principle Model.} Link-related traffic flow is the most influential factor affecting the degree of association. When the associated traffic volume of the road section is large, the platoon can stabilize the driving between adjacent intersections and maintain good continuity. According to the principle that the adjacent intersections with large traffic associated with the road section are preferentially divided into one subarea, the associated traffic within any two adjacent intersections in the area is measured and sorted, and then the initial partition plan is performed in descending order. On this basis of initial plan, the link-related traffic flow between the single intersection and the divided subarea or the subarea and the reordered subarea, and then the partition of the combined intersection is performed. The partition of the traffic subarea is not stopped until the link-related traffic is less than a certain threshold. The link-associated traffic of the subarea takes the minimum value of the associated traffic of all links. The specific partition model is as follows:

$$
\left\{\begin{array}{l}
D_{x}, D_{y} \in\left\{D_{1}, D_{2}, \ldots \ldots, D_{n}\right\}, \\
I_{i}\left(D_{x}, D_{y}\right) \in\left\{I_{1}, I_{2}, \ldots \ldots, I_{m}\right\}, \\
q_{k}\left(I_{i}, I_{j}\right) \in\left\{q_{1}, q_{2}, \ldots \ldots, q_{m-1}\right\}, \\
\operatorname{Rank}\left\{q_{1}, q_{2}, \ldots \ldots, q_{m-1}\right\}, \\
I_{j}\left(D_{x}, D_{y}\right)=0 \mid q_{k}\left(I_{i}, I_{j}\right)<Q_{\mathrm{TNS}}, \\
I_{j}\left(D_{x}, D_{y}\right)=1 \mid q_{k}\left(I_{i}, I_{j}\right) \geq Q_{\mathrm{TNS}}, \\
N_{\mathrm{TS}}=K\left(I_{1}, I_{2}, \ldots . .\right) .
\end{array}\right.
$$

In the abovementioned formula, $D_{1}, D_{2}, \ldots \ldots, D_{n}$ is $n$ intersections; $I_{1}, I_{2}, \ldots ., I_{m}$ is $m$ sections; $q_{1}, q_{2}, \ldots \ldots, q_{m-1}$ is the associated traffic flow of the sections on the $m$ sections, or the associated traffic flow of the sections; $\operatorname{Rank}\left\{q_{1}, q_{2}, \ldots \ldots, q_{m-1}\right\}$ is the sorting function, sorting the associated traffic of the sections in descending order; $Q_{\mathrm{TNS}}$ is the separation threshold of the associated traffic flow of the link; $K\left(I_{1}, I_{2}, \ldots.\right)$ is the number of subareas calculation function of the subarea division scheme; and $N_{\mathrm{TS}}$ is the total number of subareas.

When the length of the link is short, the two intersections are generally divided into one traffic subarea. And the optimal link length of the traffic subarea is between $0-800$ meters. It means that when the distance between adjacent intersections is less than 800 meters, the two intersections are divided into traffic subareas. If the link length is greater than $800 \mathrm{~m}$, the adjacent intersections are divided into different traffic subareas. If the link length is within 800 meters, there is a traffic attraction point with a large traffic volume in the middle of the link, and there is a large influence on the link traffic volume or there are other factors affecting the platoon on the link, it is generally not divided into one traffic subarea. The specific partition model is as follows: 


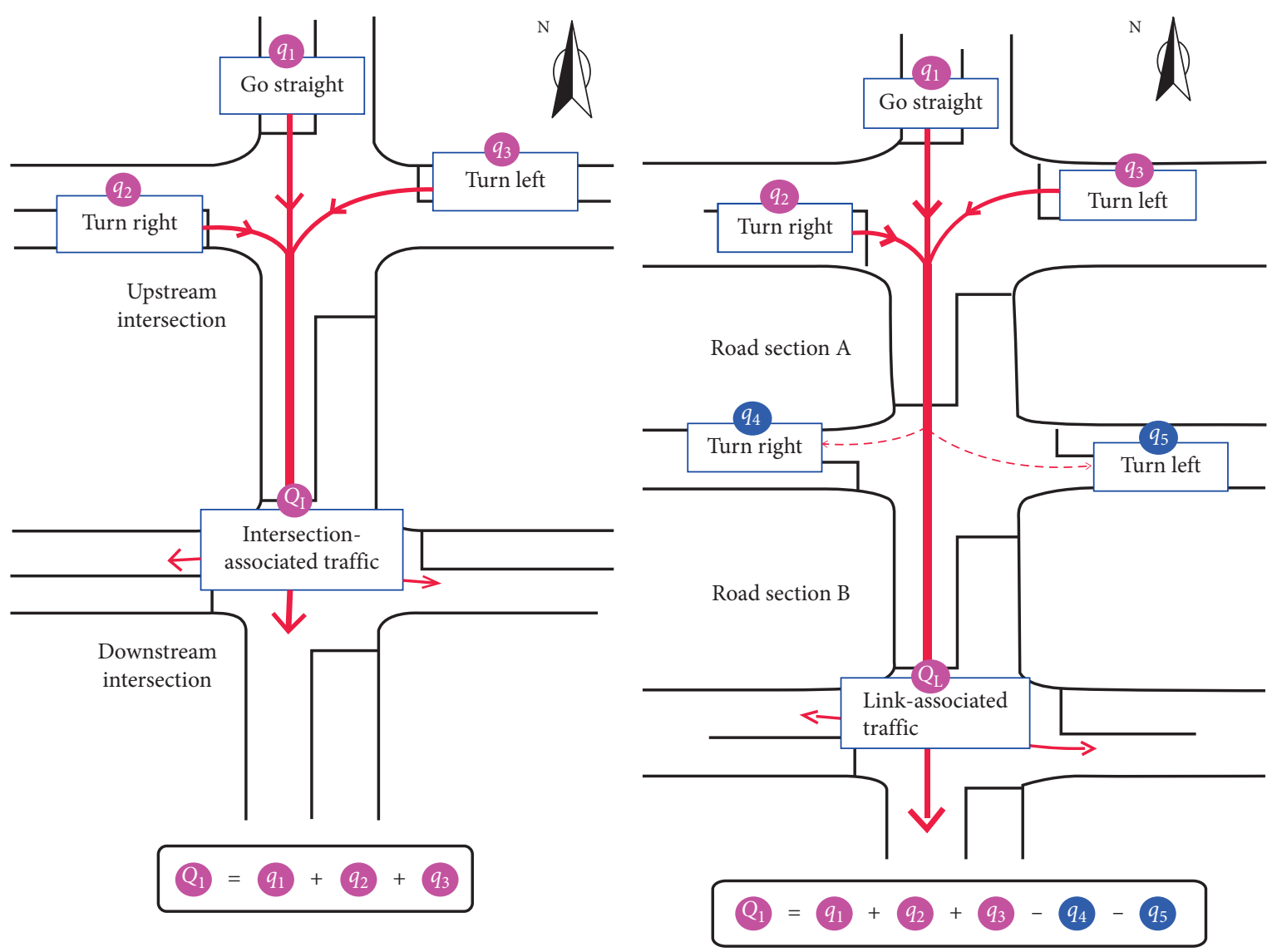

(a)

(b)

Figure 1: Associated traffic of (a) intersection and (b) link.

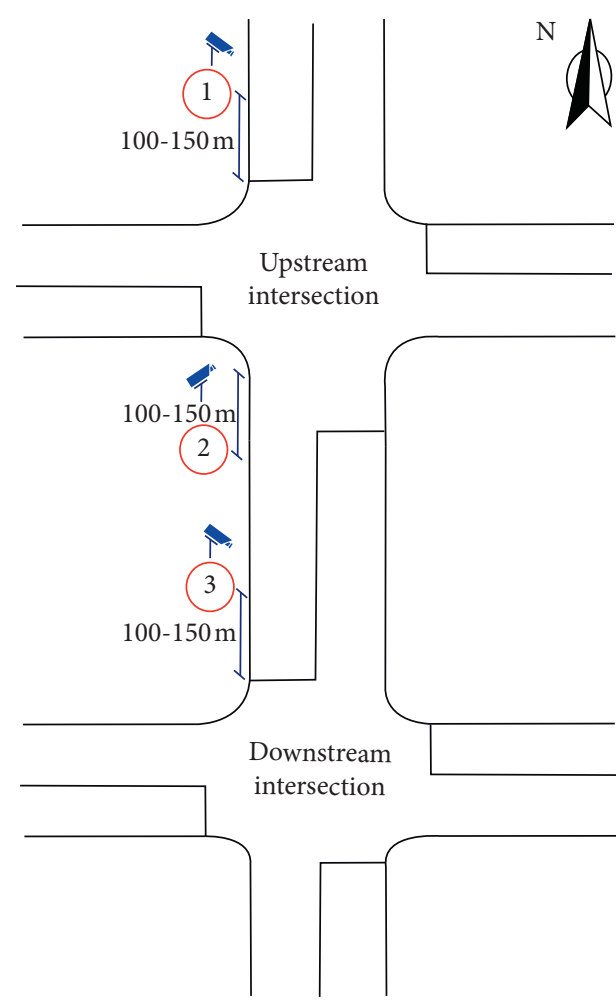

(a)

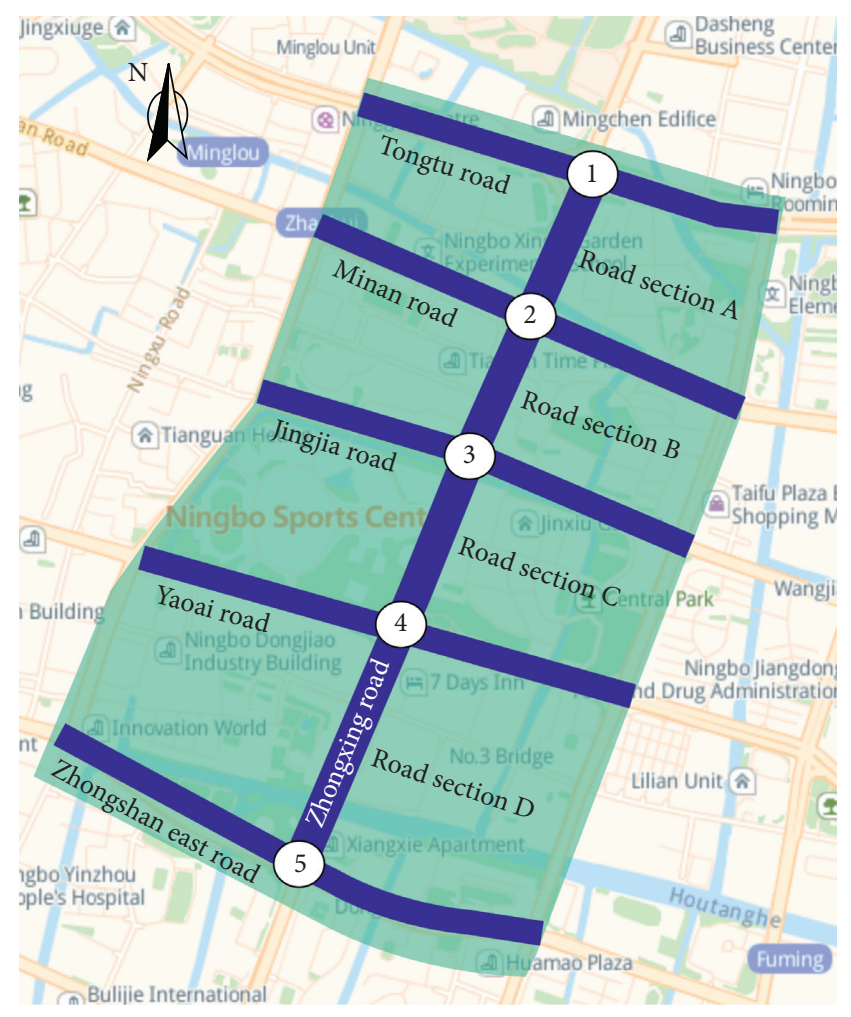

(b)

Figure 2: (a) Cameras layout. (b) Data collection area. 


$$
\left\{\begin{array}{l}
D_{x}, D_{y} \in\left\{D_{1}, D_{2}, \ldots \ldots, D_{n}\right\} \\
l_{i}\left(D_{x}, D_{y}\right) \in\left\{I_{1}, I_{2}, \ldots \ldots, I_{m}\right\} \\
I_{i}\left(D_{x}, D_{y}\right)=0 \mid l_{i}\left(D_{x}, D_{y}\right)<800 \\
I_{i}\left(D_{x}, D_{y}\right)=1 \mid l_{i}\left(D_{x}, D_{y}\right) \geq 800 \\
N_{\mathrm{TS}}=K\left(I_{1}, I_{2}, \ldots \ldots\right) .
\end{array}\right.
$$

In the abovementioned formula, $l_{i}\left(D_{x}, D_{y}\right)$ is the length of the link between the intersections $D_{x}$ and $D_{y}$.

Adjacent intersections with the same signal cycle length are divided into one traffic subarea. When the optimal signal cycle lengths of adjacent intersections are the same, the traffic conditions of these two intersections are similar, and it is easier to coordinate control in one traffic subarea. The specific partition model is as follows:

$$
\left\{\begin{array}{l}
D_{x}, D_{y} \in\left\{D_{1}, D_{2}, \ldots \ldots, D_{n}\right\} \\
l_{i}\left(D_{x}, D_{y}\right) \in\left\{I_{1}, I_{2}, \ldots \ldots, I_{m}\right\} \\
I_{i}\left(D_{x}, D_{y}\right)=0 \mid C_{x} \neq N C_{y} \\
I_{i}\left(D_{x}, D_{y}\right)=1 \mid C_{x}=N C_{y} \\
N_{\mathrm{TS}}=K\left(I_{1}, I_{2}, \ldots . .\right) .
\end{array}\right.
$$

In the abovementioned formula, $\mathbf{N}$ is an integer multiple of $1 / 2$ or an integer and $C_{x}$ is the Cycle length of the intersection $x$.

3.2.2. Association Degree Partition Model. On the basis of the initial partition scheme, the boundary of the traffic subarea is determined by the correlation degree partition model, and then the optimal subarea division scheme is formed. The traffic subarea partition model is based on the model proposed by $\mathrm{Lu}$ [21]; however, the assumption constraints are improved and the association degree model proposed in this paper is established. The specific model is as follows:

$$
\left\{\begin{array}{l}
D_{x}, D_{y} \in\left\{D_{1}, D_{2}, \ldots \ldots, D_{n}\right\} \\
I_{i}\left(D_{x}, D_{y}\right) \in\left\{I_{1}, I_{2}, \ldots \ldots, I_{m}\right\} \\
R_{i}\left(D_{x}, D_{y}\right)=\frac{1}{1+|2.6-0.06 \times q+0.002 \times l|}, \\
I_{i}\left(D_{x}, D_{y}\right)=0 \mid R_{i}\left(D_{x}, D_{y}\right)<R_{\mathrm{TNS}}, \\
I_{i}\left(D_{x}, D_{y}\right)=1 \mid R_{i}\left(D_{x}, D_{y}\right) \geq R_{\mathrm{TNS}}, \\
R_{A i}=\frac{\sum_{j=1}^{N_{A}} R_{i}\left(D_{x}, D_{y}\right)}{N_{A}}, R_{\mathrm{TA}}=\sum_{i=1}^{N_{\mathrm{TS}}} R_{A i}, N_{\mathrm{TS}}=K\left(I_{1}, I_{2}, \ldots . .\right), P=F\left(E_{1}, E_{2}, \ldots . .\right), R_{A i}>R_{\mathrm{TMS}} .
\end{array}\right.
$$

In the abovementioned formula, $R_{i}\left(D_{x}, D_{y}\right)$ is the degree of association of intersections, $R_{\mathrm{TNS}}$ is the separation threshold of adjacent intersections, $R_{\mathrm{TMS}}$ is the threshold of separation of multiple intersections, $R_{A i}$ is the intersection degree of multiple intersections and its algorithm is the arithmetic mean of the degrees of association of all adjacent intersections in the set, $R_{\mathrm{TA}}$ is the total degree of association of the region, $E_{1}, E_{2} \ldots \ldots$ is the evaluation index variable of the sub-area division scheme, $F\left(E_{1}, E_{2} \ldots \ldots\right)$ is the evaluation function of the subarea division scheme, and $P$ is the evaluation value of the subarea division scheme.

3.3. Traffic Subarea Partition Process. In the actual partition of the traffic subarea, to use the traffic subarea partition model to get the best traffic subarea partitioning scheme requires designing a complete coordinated control traffic subarea partitioning process and scheme evaluation method. 
This paper designed a complete flow subarea division process, as shown in Figure 3.

The reason why the separation threshold in the abovementioned flow chart is 0.2 is as follows. Firstly, in general, it is considered that when the average traffic volume is 0.8 times greater than the traffic capacity, traffic congestion is caused. Secondly, in the traffic impact assessment, when the $\mathrm{V} / \mathrm{C}$ ratio is greater than 0.8 , traffic congestion is considered. The optimal partitioning scheme should satisfy the optimal evaluation index of the traffic subarea partitioning scheme. The specific evaluation index includes the green wave bandwidth and the continuous green wave transit time in the late green wave design.

\section{Model Validation and Discussion}

4.1. Relevance Model. In order to verify the reliability of the proposed model, the coordination coefficient in the SYNCHRO software is selected for verification. SYNCHRO's coordination coefficient [22] comprehensively considers the four adjustment factors of travel time, platoon, traffic flow, and signal period length. The considerations are comprehensive and have certain reference value. The specific model is as follows:

$$
\mathrm{CF}=\operatorname{Max}(C F 1, C F 2)+\mathrm{Ap}+\mathrm{Av}+\mathrm{Ac} .
$$

In the abovementioned formula, $C F 1$ is the primary coordination factor for travel time; $C F 2$ is the primary coordination factor for road segment density; Ap is the platoon adjustment factor; Av is the flow adjustment factor; and Ac is the signal period length adjustment factor. The calculation formulas of each coordination factor are as follows:

$$
\left\{\begin{array}{l}
C F 1=100-(\text { time }-4) \times 100 \div 76, \\
C F 2=100 \times q \div(n \times \text { LinkDist } \div \text { VL }), \\
\text { Ap }=10-(100-\text { PulseFactor }) \times 30 \div 55, \\
\text { PulseFactor }=\left(v_{30}+v_{60}\right) /(2 * v c) * 100 \%, \\
\text { Av }=\left\{\begin{array}{l}
\frac{\left(v_{2}-700\right)}{50}, v_{2}<1200, \\
\frac{\left(v_{2}-200\right)}{100}, v_{2}<2200, \\
\text { Ac }=-\frac{\left(\sum C_{i}\right)}{2},
\end{array}\right.
\end{array}\right.
$$

where $q$ is average traffic; $n$ is lanes in through the lane group; LinkDist is link distance minus 150 feet $(45 \mathrm{~m})$ for the space in intersection and extra space; VL is average vehicle length entered in network settings; PulseFactor is the proportion of upstream traffic in the busiest part of cycle; $v_{30}$ is the volume of traffic arriving during the busiest $30 \%$ of the cycle; $v_{60}$ is the volume of traffic arriving during the busiest $60 \%$ of the cycle; vc is the volume per cycle; $v_{2}$ is the sum of two ways volume on link (vph); and $C_{i}$ is the amount to increase cycle length for coordination.

The data of 8 consecutive intersections from the intersection of Ximenwai Street and Xinxi Road in the Miyun District of Beijing were collected to verify the correlation model proposed in this paper, as shown in Figure 4. The survey data are used to calculate the correlation and CF values of adjacent intersections within the survey area, as shown in Table 1 .

The signal timing plans of adjacent intersections in the Gulou W Street, Miyun District, Beijing, are obtained from practical survey data (the number of these 8 intersections is numbered from $\mathrm{ABCDEFGH}$ in the eastbound direction), as shown in Table 1. The traffic volumes at the PM peak hour of the eight intersections are shown in Figure 5. Using the CF value and the relevance model proposed in this paper, the correlation degrees of each adjacent intersection in the survey range are calculated. The calculation results and the correlation results of the two models are shown in Table 2 and Figure 6.

4.2. Traffic Subarea Partition Model. In order to verify the reliability of the traffic subarea partition model, the division methods from SYNCHRO and Chang were selected for comparison. The traffic simulation process was carried out with SimTraffic. According to Synchro Studio 8.0, when the CF value is greater than 80 , adjacent intersections should be coordinated in isolated traffic subarea. According to the principle of automatic intersection of adjacent intersections proposed by Chang [3], the separation threshold of adjacent intersections is set to 0.35 . When the degree of association of adjacent intersections is greater than or equal to 0.35 , the two adjacent intersections are suitable for coordinated control. According to the subarea division methods mentioned above, the separation threshold of adjacent intersections is set to 0.2 . The results of each program divisions are shown in Table 3 and Figure 7.

When the abovementioned three division schemes are matched, only the third division scheme can get green wave with design wave speed of $40 \mathrm{~km} / h$, and the design time interval diagram of green wave is shown in Figure 8.

In order to evaluate the operation of the eight intersections of the road section according to the three division schemes, the simulation is carried out by SimTraffic. The specific evaluation results are shown in Table 4 .

\subsection{Discussion}

4.3.1. Verification Results of the Intersection Correlation Model. According to the results of Figure 7, the trend of the correlation degree obtained by the new intersection correlation model and the SYNCHRO coordination coefficient is generally consistent. When the coordination coefficient 


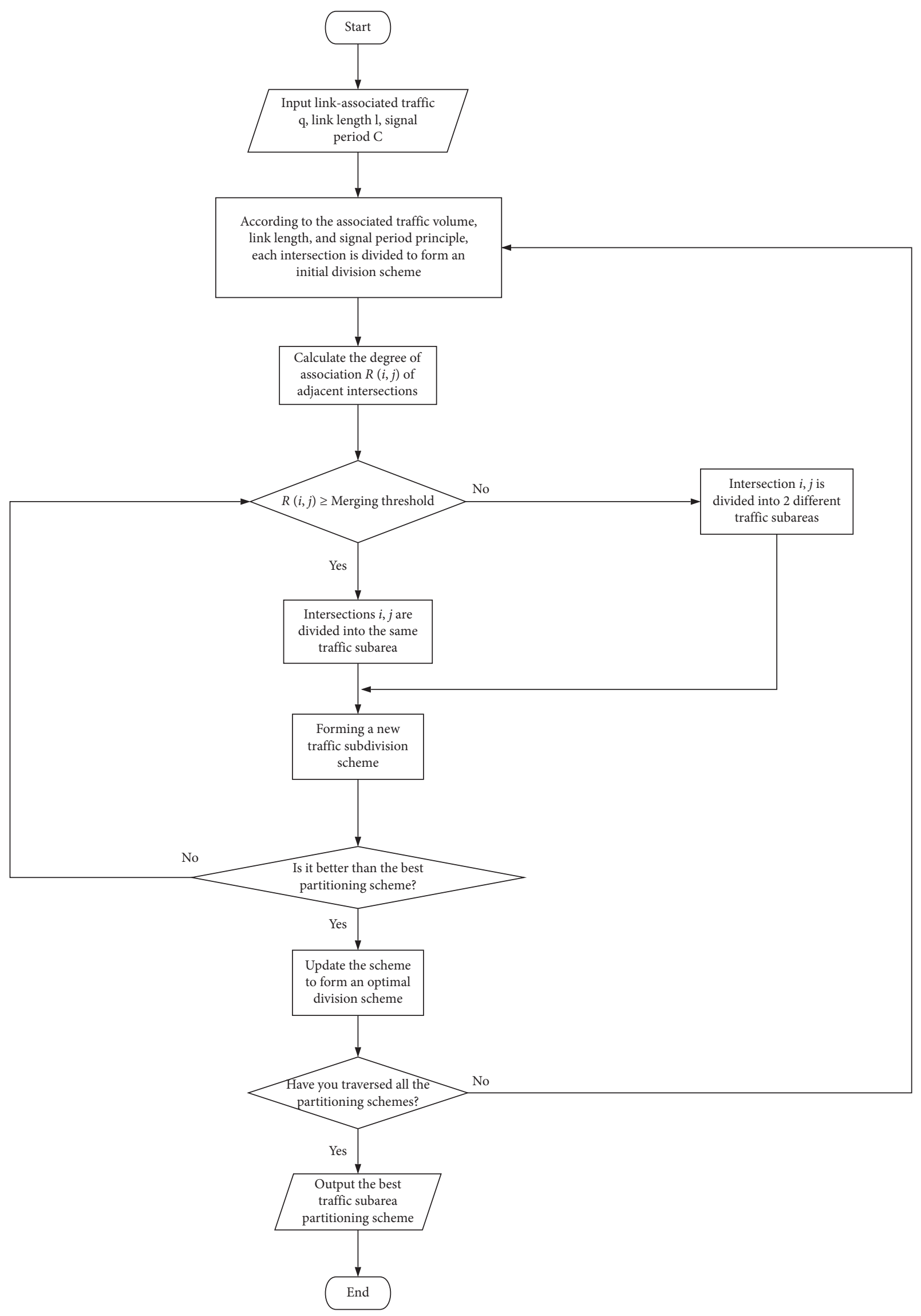

Figure 3: Traffic subarea partition flow chart. 


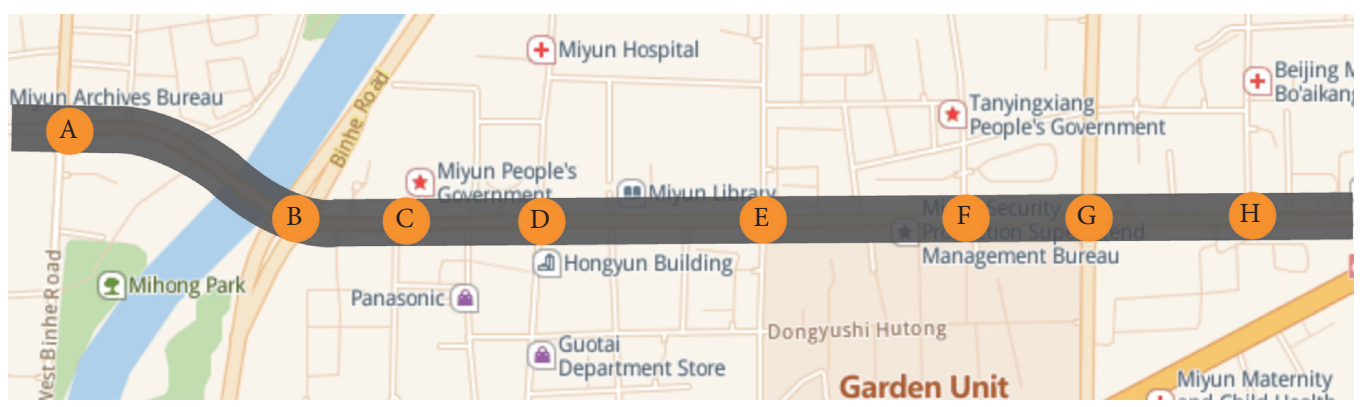

Figure 4: Study area of model application, Gulou W St, Miyun District, Beijing

TABLe 1: Basic information of Gulou W St, Miyun District, Beijing.

\begin{tabular}{|c|c|c|c|c|c|}
\hline $\begin{array}{l}\text { Intersection } \\
\text { number }\end{array}$ & Name of intersection & $\begin{array}{c}\text { Types of } \\
\text { intersections }\end{array}$ & $\begin{array}{l}\text { The length of the road } \\
\text { section }(\mathrm{m})\end{array}$ & $\begin{array}{l}\text { Signal period } \\
\text { (seconds) }\end{array}$ & $\begin{array}{c}\text { Phase } \\
\text { number }\end{array}$ \\
\hline A & Ximen outer St, Xinxi Rd & Crossbonding & - & 134 & 4 \\
\hline B & Gulou W St, Binhe Rd & Oblique $X$ type & 520 & 71 & 2 \\
\hline C & $\begin{array}{c}\text { Gulou W St, Miyun District } \\
\text { government }\end{array}$ & T type & 245 & 70 & 2 \\
\hline $\mathrm{D}$ & Gulou W St, Gulou S St & Crossbonding & 230 & 144 & 4 \\
\hline $\mathrm{E}$ & Gulou W St, Xinzhong St & Crossbonding & 445 & 125 & 4 \\
\hline $\mathrm{F}$ & Gulou W St, Yucai Rd & T type & 415 & 85 & 2 \\
\hline G & Gulou W St, Xindong Rd & Crossbonding & 230 & 126 & 4 \\
\hline $\mathrm{H}$ & Gulou W St, Xinggong Rd & T type & 305 & 72 & 2 \\
\hline
\end{tabular}

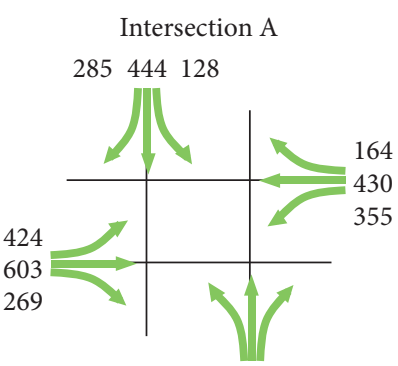

211436494

Intersection $\mathrm{E}$

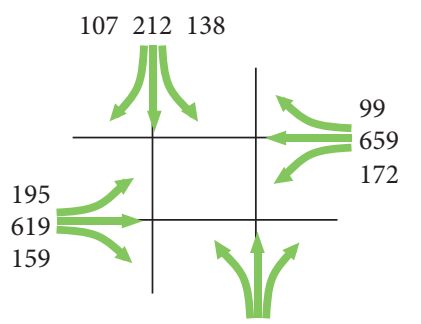

13924793

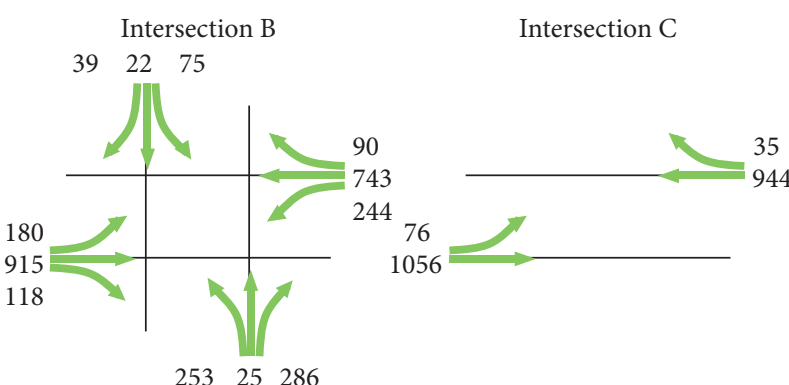

$253 \quad 25286$

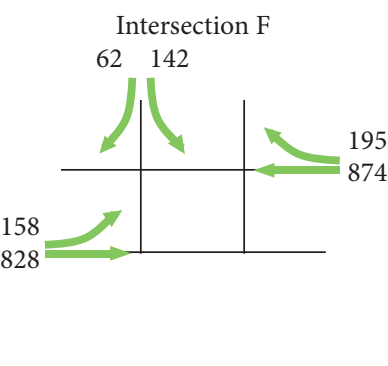

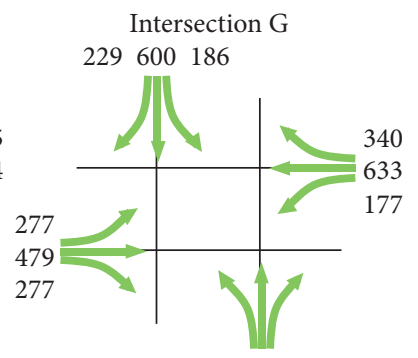

244633216

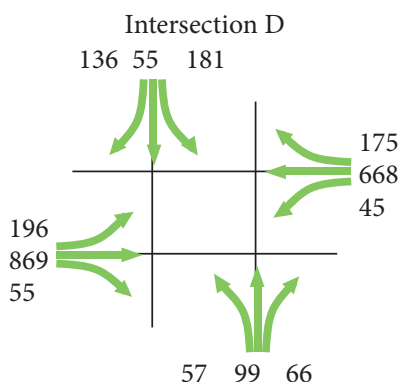

$5799 \quad 66$

FIgURE 5: Evening peak traffic flow at 8 intersections of Gulou W St.

TABLE 2: Relevance model validation and comparison.

\begin{tabular}{lccccc}
\hline $\begin{array}{l}\text { Road section } \\
\text { name }\end{array}$ & $\begin{array}{c}\text { Linkage related traffic } \\
(\mathrm{pch} / \mathrm{C})\end{array}$ & Link length $(\mathrm{m})$ & Signal cycle length $(\mathrm{s})$ & $\begin{array}{c}\text { Coordination coefficient } \\
(\text { SYNCHRO) }\end{array}$ & Correlation degree \\
\hline $\mathrm{AB}$ & 15.52 & 520 & 110 & 72 & 93 \\
BC & 16.18 & 240 & 70 & 102 & 0.27 \\
$\mathrm{CD}$ & 29.00 & 245 & 112 & 82 & 0.32 \\
$\mathrm{DE}$ & 32.76 & 445 & 156 & 80 & 0.43 \\
EF & 26.63 & 410 & 85 & 103 & 0.40 \\
FG & 31.54 & 230 & 170 & 76 & 0.35 \\
GH & 14.56 & 310 & 54 & 0.46 \\
\hline
\end{tabular}




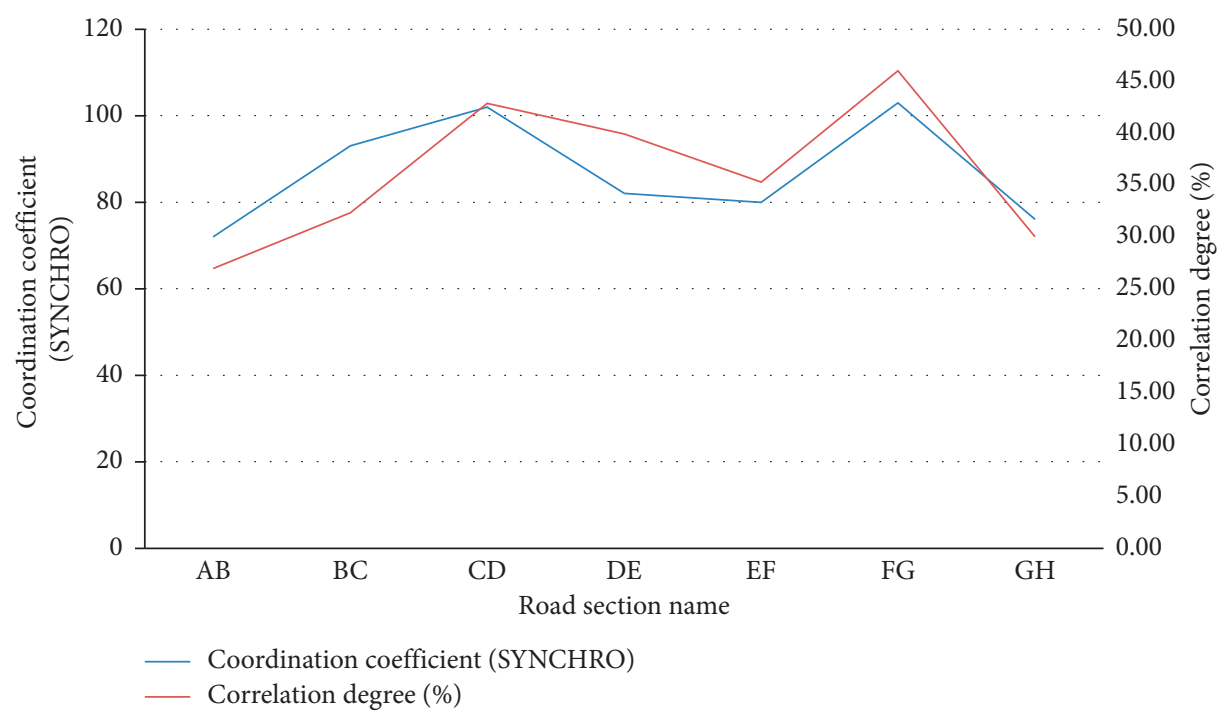

FIgURE 6: Trend of correlation model and coordinatability factor.

TABLE 3: Comparison of three subarea partition scheme.

Subarea partitioning scheme

SYNCHRO subarea division scheme 1
Number of subzones

Number of subzones

Subarea contains intersections

\begin{tabular}{lc}
1 & A \\
2 & BCDEFG \\
3 & $\mathrm{H}$ \\
1 & $\mathrm{~A}$ \\
2 & $\mathrm{~B}$ \\
3 & $\mathrm{CDEFG}$ \\
4 & $\mathrm{H}$ \\
\hline
\end{tabular}

$1 \quad$ ABCDEFGH

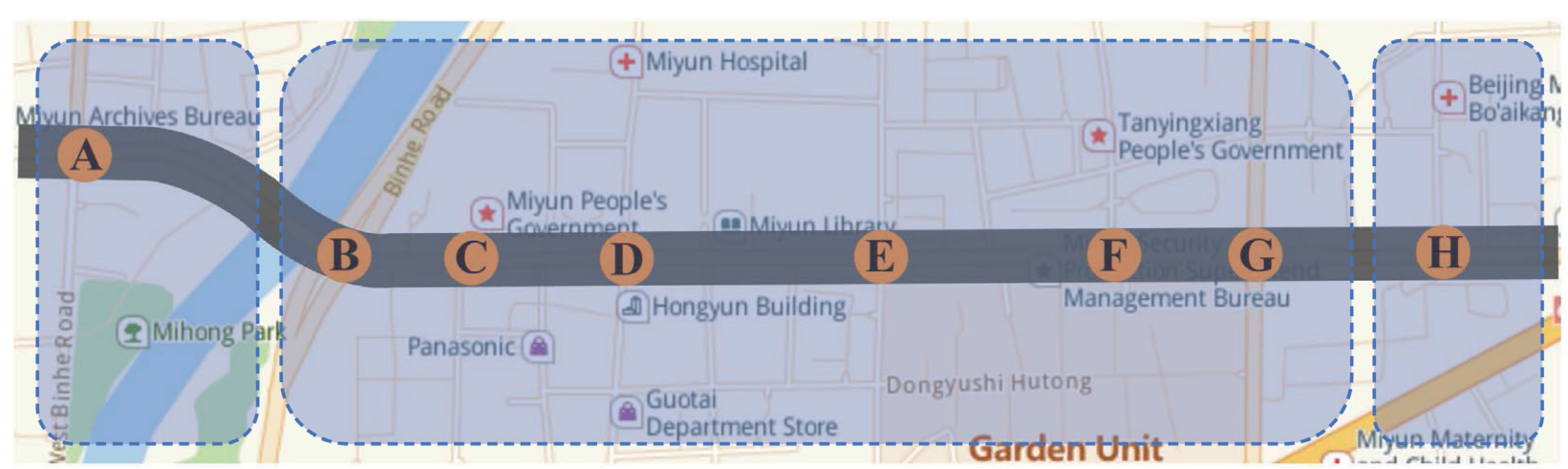

(a)

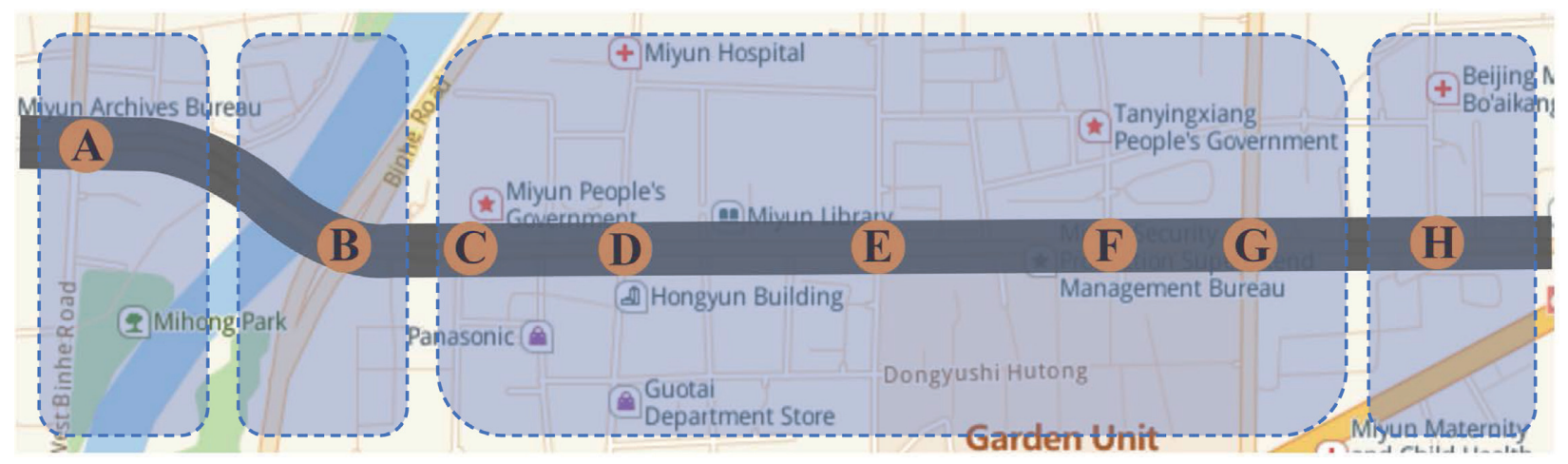

(b)

Figure 7: Continued. 


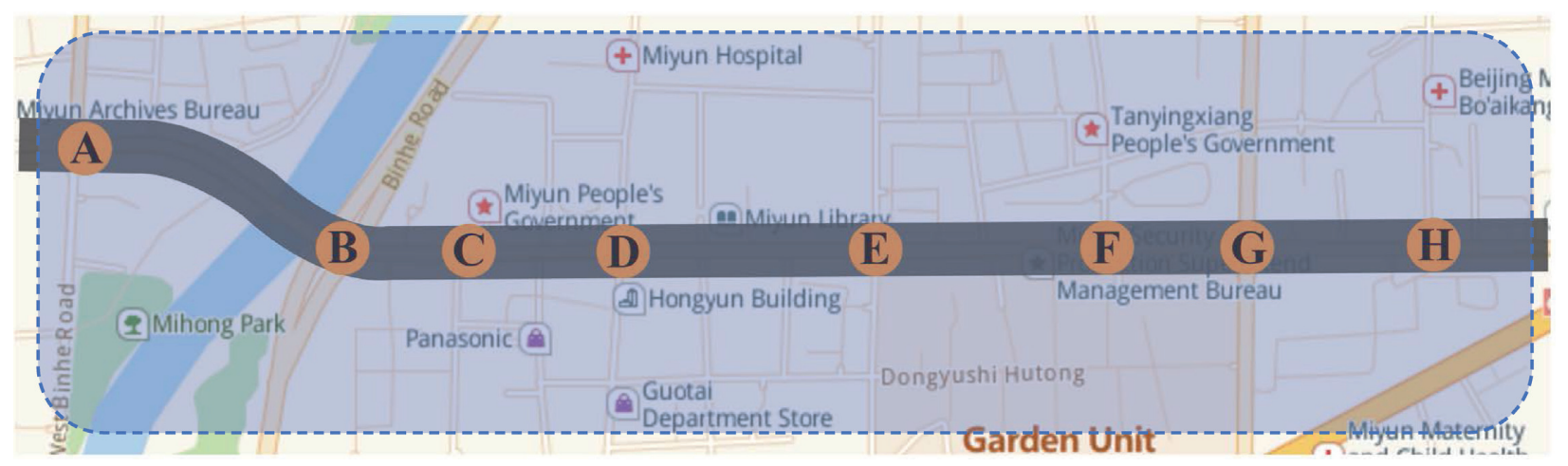

(c)

FIGURE 7: Schematic diagram of three control subarea partitioning methods.

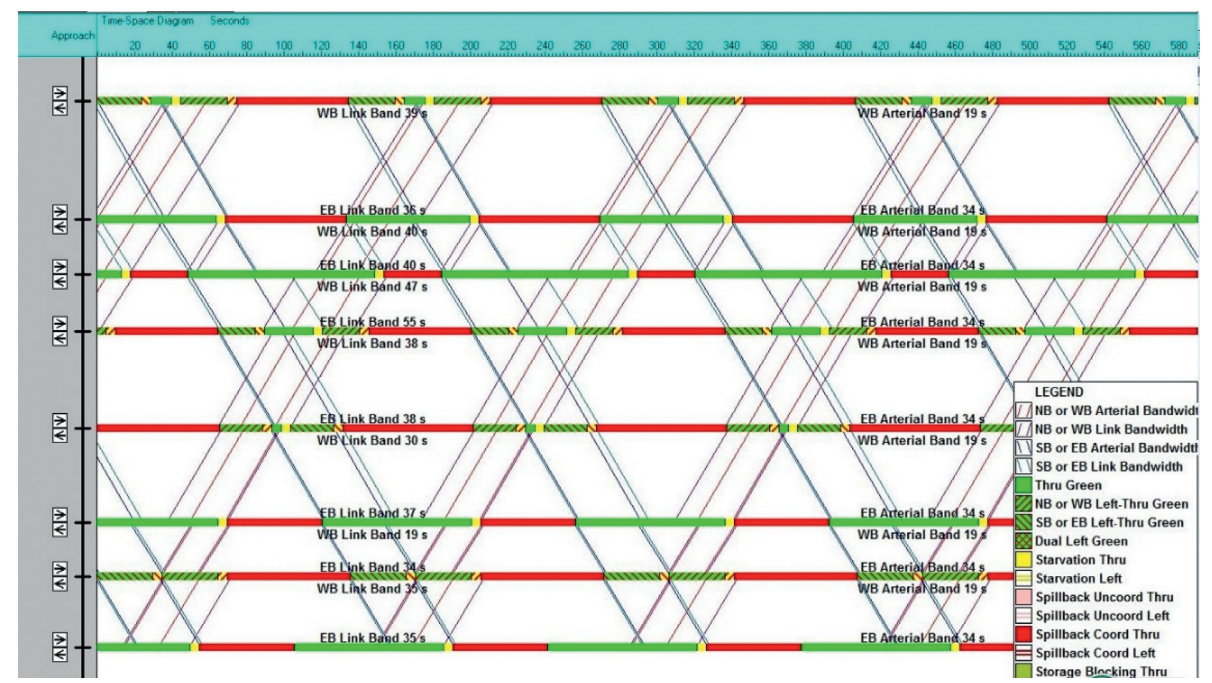

FIGURE 8: Time-space diagram for this arterial road. (a) Division based on SYNCHRO coordination coefficient, Scheme 1. (b) Subarea division based on Chang's method of subarea division, Scheme 2. (c) Subarea division method proposed in this paper, Scheme 3.

TABLE 4: Comparison of performance indexes of three subarea division schemes.

\begin{tabular}{lcccc}
\hline Evaluation index & Status quo & CF value subarea division & 0.35 relevance subarea division & 0.20 relevance subarea division \\
\hline Total delay (h) & 86.5 & 76.3 & 74.5 & 73.5 \\
Car delay (s) & 204.1 & 168.4 & 163.5 & 160.4 \\
Total number of stops & 3047 & 2834 & 2889 & 2754 \\
Schedule time & 112 & 104.6 & 103.1 & 102.2 \\
\hline
\end{tabular}

increases, the correlation degree calculated by the new correlation degree model also increases. The connection of adjacent intersections is closer, and the degree of association is higher. The reliability of the relevance degree model is verified. Because the calculation formula of the coordination coefficient CF is complex, the required data is difficult to obtain; however, the coordination coefficient formula is simple, from the empirical data. Therefore, the relevance model of this paper is considered more practical.

4.3.2. The Verification Result of the Traffic Control Subarea Partition Model. According to the results in Table 2, these eight signalized intersections within the research range are partitioned into three subareas, four subareas, and one separately using those three methods. There are three partition schemes for subareas. The first two partition schemes have more isolated intersections as separate independent subareas. On the one hand, the situation is more complicated when coordinated control is performed, and the signal timing optimization is difficult. According to the partition scheme proposed in this paper, the coordination control is the simplest and the coordination effect is the best. As shown in Figure 8, the green wave band with the designed wave velocity of $40 \mathrm{~km} / \mathrm{h}$ is obtained based on the partition scheme 3. The eastbound through movement bandwidth is 34 seconds and westbound through movement bandwidth is 19 seconds, and the total green bandwidth is 53 seconds. The 
average link bandwidth of eastbound through movement is 39 seconds, and the average link bandwidth of westbound through movement is 35 seconds.

4.3.3. Simulation Evaluation Results. Signal timing optimization and coordinated control are performed on the intersections of the three division schemes. According to the simulation results in Table 3, in the subarea partition scheme based on the 0.20 correlation degree proposed in this paper, the total delay, average vehicle delay, total number of stops, and travel time of the entire road network are minimized and the evaluation indexes are optimal. Compared with the current situation, the total vehicle delay in the intersections is reduced by $15.03 \%$, the average vehicle delay is reduced by $21.41 \%$, the total number of stops is reduced by $9.62 \%$, and the travel time is shortened by $8.75 \%$. The coordinated control of the partition scheme obtained by the traffic control subarea partitioning model proposed in this paper can greatly improve the traffic efficiency of intersections and help alleviate urban traffic congestion.

\section{Conclusions}

Based on the analysis of survey data, this paper studied the key technologies of traffic subarea partition, and the main research results and innovations are as follows:

(1) The vehicle's discreteness is measured by the headway. The correlation degree $R$ is defined quantitatively so that the traffic subarea partition is more intuitive and simple. The calculation model of the intersection correlation degree based on the link-related traffic and the length of the link is constructed. It is verified that the model is feasible and effective, which lays a foundation for the partition of the traffic subareas.

(2) Based on the intersection degree model, combined with the traffic subarea partition principle, partition criteria, constraints, evaluation indicators, and coordination control objectives, the metricized traffic subarea partition model is established, and the effective regulation of traffic subarea partition is realized.

(3) Based on these advantages and disadvantages of the existing traffic subarea division process, combined with the results of this paper, a set of scientific and practical traffic subarea division process is designed to absorb the advantages and avoid the shortcomings.

(4) The research results of this paper are studied from the actual traffic data and applied to the data, which is more practical and more valuable.

In the future study, the traffic path and the OD matrix in the region should be considered for the method of dividing subarea to achieve the optimal overall region.

\section{Data Availability}

The data used to support the findings of this study are available from the corresponding author upon request.

\section{Disclosure}

Additionally, this manuscript was presented (submission just for presentation, not for publication) in TRB Annual Meeting 2020 Poster Session AHB25 (paper number is 2001254).

\section{Conflicts of Interest}

The authors declare they have no conflicts of interest.

\section{Acknowledgments}

This study was supported by project “The Fundamental Research Funds for the Central Universities (no. 2019JBM028)".

\section{References}

[1] D. Husch and J. Albeck, Synchro 6: Traffic Signal Software, User Guide, Trafficware, Ltd, Albany, Calif, 2003.

[2] H. N. Yagoda, E. H. Princiole, and C. E. Vick, "Subdivision of signal systems into control areas," Traffic Engineering - Institute of Transportation Engineers, vol. 43, no. 12, pp. 42-45, 1973.

[3] E. C. P. Chang, How to Decide the Interconnection of Isolated Traffic Signals, ACM, New York, NY, USA, 1985.

[4] Highway Capacity Manual 1987(HCM 1987), National Research Council, Washington, DC, USA, 1987.

[5] NAC, Traffic Control Devices Handbook [M], Federal Highway Administration, Washington, DC, USA, 1975.

[6] R. Li and P. Fan, "Correlativity-based traffic control sub-area dynamic division method research [EB/OL]," http://www. paper.edu.cn.

[7] K. Lu, J. Xu, and S. Zheng, "Correlation degree analysis of neighboring intersections and application," Journal of South China University of Technology (National Science Edition), vol. 37, no. 11, pp. 37-42, 2009.

[8] H. Duan, Z. Li, Yi Zhang, and J. Hu, "Dynamic subdivision of road network into coordinated control regions," Journal of Jilin University (Engineering and Technology Edition), vol. 37, no. 11, pp. 37-42, 2009.

[9] J. Yang, X. Guo, L. I. Yan et al., "Modeling route correlation degree of urban signalized intersection group," Journal of Transportation System Engineering and Information Technology, vol. 12, no. 1, pp. 55-62, 2012.

[10] J.-min Xu, Y. Xiao-wen, B.-bin Jing, and Yu-jun Wang, "Dynamic network partitioning method based on intersections with different degree of saturation," Journal of Transportation Systems Engineering and Information Technology, vol. 4, no. 17, pp. 145-152, 2017.

[11] X. W. Lin, Study on Urban Road Network Division Method Based on Road Network Correlative Degree Analysis, Jilin University, Changchun, China, 2017.

[12] L. Y. Zeng, Research on Dynamic Partitioning Method of Traffic Control Subarea for Different Traffic Conditions, South China University of Technology, Guangzhou, China, 2017.

[13] R. J. Walinchus, "Real-time network decomposition and subnetwork interfacing," Highway Research Record, pp. 20-28, National Academy of Science, Washington, DC, USA, 1971.

[14] FHWA, Manual on Uniform Traffic Control Devices for Streets and Highways, Federal Highway Administration, Washington, DC, USA, 1978. 
[15] P. R. Lowrie, "The sydney coordinated adaptive traffic systemprinciples, methodology, algorithms," in Proceedins of the International Conference on Road Traffic Signaling, Institution of Electrical Engineers, London, UK, pp. 67-70, 1982.

[16] W. M. Zhao, Research on Several Urban Regional Traffic Control Technical, Zhejiang University, Hangzhou, China, 2014.

[17] W. T. Zhao, Time-of-Day Breakpoints Identification and Traffic Network Partition Methods for Traffic Control, Zhejiang University, Hangzhou, China, 2013.

[18] D. Xia, B. Wang, Y. Li, Z. Rong, and Z. Zhang, "An efficient MapReduce-based parallel clustering algorithm for distributed traffic subarea division," Discrete Dynamics in Nature and Society, vol. 2015, Article ID 793010, 18 pages, 2015.

[19] Y. M. Xie, "Research on the method of traffic control sub-area partition," Journal of Yancheng Institute of Technology (Natural Science Edition), vol. 31, no. 1, pp. 74-78, 2018.

[20] W. Xianyu, H. Peifeng, and Y. Zhenzhou, "Link-based signalized arterial progression optimization with practical travel speed," Journal of Applied Mathematics, vol. 2013, Article ID 197313, 13 pages, 2013.

[21] K. Lu, Research on the Foundational Theory and Key Techniques or Coordinate Signal Control in Urban Traffic Network, South China University of Technology, Guangzhou, China, 2010.

[22] Trafficware, Ltd, Synchro Studio 8 User Guide, Trafficware, Ltd, PO Box 499 Sugar Land, USA, 2011. 